\title{
Assessments of the Value of New Interventions Should Include Health Equity Impact
}

\author{
Jeroen P. Jansen ${ }^{1,3}$ (1) $\cdot$ Thomas A. Trikalinos ${ }^{2} \cdot$ Kathryn A. Phillips ${ }^{1,3}$
}

Accepted: 16 January 2022 / Published online: 3 March 2022

(c) The Author(s) 2022

\begin{abstract}
A formal evaluation of the health equity impact of a new intervention is hardly ever performed as part of a health technology assessment to understand its value. This should change, in our view. An evidence-based quantitative assessment of the health equity impact can help decision makers develop coverage policies, programme designs, and quality initiatives focused on optimizing both total health and health equity given the treatment options available. We outline the conceptual basis of how a new intervention can impact health equity and adopt distributional cost-effectiveness analysis based on decision-analytic models to assess this quantitatively, using a newly US FDA-approved drug for Alzheimer's disease (aducanumab) as an example. We argue that gaps in the evidence base for the new intervention, for example, due to limited clinical research participation among racial and ethnic minority groups, do not preclude such an evaluation. Understanding these uncertainties has implications for fair pricing, decision making, and future research. If we are serious about population-level decision making that not only is focused on improving total health but also aims to improve health equity, we should consider routinely assessing the health equity impact of new interventions.
\end{abstract}

\section{Introduction}

The US FDA approved aducanumab for early Alzheimer's disease (AD) in June 2021 [1]. The excitement about this possible first disease-modifying therapy for AD is complicated by its uncertain benefits, potential risks, and costs, thereby rekindling long-standing questions about what constitutes a valuable new drug in the public's eye. The COVID-19 pandemic laid bare the health disparities in access to quality

Jeroen P. Jansen

jeroen.jansen@ucsf.edu

Thomas A. Trikalinos

thomas_trikalinos@brown.edu

Kathryn A. Phillips

kathryn.phillips@ucsf.edu

1 Department of Clinical Pharmacy, Center for Translational and Policy Research on Personalized Medicine (TRANSPERS), University of California, San Francisco, 490 Illinois Street, Rm 32M, San Francisco, CA 94158, USA

2 Departments of Health Services, Policy, and Practice and Biostatistics, Center for Evidence Synthesis in Health, Brown University School of Public Health, Providence, RI, USA

3 Philip R. Lee Institute for Health Policy Studies, University of California, San Francisco, CA, USA care, resources, and outcomes among racial and ethnic minority populations, socioeconomically vulnerable individuals, and populations in rural areas, which have also been raised by health equity scholars for decades [2-6]. Post-2020, in the backdrop of renewed and keener scrutiny of health equity issues, the consequences of an intervention across these population subgroups and whether it attenuates or perpetuates disparities in health outcomes should come to the forefront. However, to date, a formal health equity impact evaluation of a new intervention is hardly ever performed as part of a health technology assessment (HTA). The lack of information about the expected impact of aducanumab on the significant and persistent health outcome disparities across racial groups in $\mathrm{AD}$ is a case in point $[7,8]$. This should change, in our view. An evidence-based quantitative assessment of the health equity impact of a new medical intervention can help decision makers develop coverage policies, programme designs, and quality initiatives focused on optimizing both total health and health equity given the treatment options available. We outline conceptually how a new intervention can impact on health equity, and we use distributional cost-effectiveness analysis (DCEA) based on decision-analytic models to assess this in a quantitative fashion despite evidence challenges, using aducanumab as an example. 


\section{Key Points for Decision Makers}

Cost-effectiveness evaluations are part of health technology assessment of new interventions to inform efficient use but do not provide information to guide policy objectives related to health equity.

Distributional cost-effectiveness analysis is an intuitively appealing extension of conventional cost-effectiveness analysis to quantify health equity impacts and facilitate potential trade-offs between improving total health and health equity.

Gaps in the evidence base for a new intervention, for example because of limited clinical research participation among racial and ethnic minority groups, do not automatically render distributional cost-effectiveness analysis moot, futile, or vacuous. Employing a decisionmodelling approach provides the framework to evaluate, understand, and communicate the implications of this uncertainty on health equity impact and estimates of value, and contributes to more honest policy discussions.

\section{Improving Overall Health While Satisfying Notions of Distributional Fairness}

We adopt the World Health Organization definition of health equity as the absence of unfair avoidable or remediable differences in health among population groups defined socially, economically, demographically, or geographically [9]. A thus-defined state of health equity satisfies a notion of distributional fairness in attained outcomes and generally implies the need to minimize the adverse impacts of societal, economic, demographic, and geographic determinants of health on marginalized groups [10]. Achieving health equity and optimizing overall health are related but distinct objectives: One can improve health outcomes on average while worsening health equity gaps, as has been repeatedly and persistently demonstrated in many health domains. And, trivially, one could achieve health equity by worsening health outcomes for everyone to a common lowest level, which reduces inequity at the cost of worsening overall health. These observations have important connotations for HTAs, which assess the value of new health technologies (diagnostics, treatments, services) with respect to whether they improve average health enough for their cost, but without explicitly considering distributional fairness, that is, whether they attenuate or accentuate health outcome inequities.

\section{Impact of a New Intervention on Inequality in Health Outcomes}

Both health outcomes and costs need to be considered in the evaluation of the health equity impact of a new intervention. Specifically, a new intervention that is effective will attenuate or exacerbate inequality in health outcomes in the target patient population of interest, and therefore positively or negatively impact health equity, if differences exist in (1) baseline event or outcome probabilities, (2) its effectiveness, or (3) accessibility or uptake between its racial, economic, demographic, or geographic subgroups. For the remainder of this paper, we label these 'social subgroups'. Differences in accessibility or uptake of a new intervention can be caused not only by disparities in insurance coverage or high patient co-payments but also by other behavioural, social-cultural, and healthcare system factors of influence at the individual, interpersonal, community, or societal level [11].

New interventions that are expensive may also have negative health consequences for individuals other than the target patient population, for whom healthcare expenditure may decline or insurance premiums may increase to offset the extra costs of the new intervention. How large is the opportunity cost of expensive interventions? Recent simulations among US people who discontinue versus continue their insurance coverage when premiums change estimate the value of health forgone at \$US100,000 per quality-adjusted life-year (QALY) [12]. Health opportunity costs may not be equally distributed across income and wealth strata, and often across racial groups, thereby further impacting on disparities in population health outcomes with the use of a new intervention for which the health outcomes do not warrant the costs.

We can use various inequality metrics or indices to quantify the dissimilarity of attained outcomes across social subgroups. We are careful to distinguish the concept of outcome inequity and our measurement of it: we use the word 'inequality' to refer to an explicit quantification, and the term 'health equity' to refer to the broader concept. For example, we use inequality metrics to describe or infer the presence or absence of outcome inequities or to quantify the health equity impact of new interventions.

\section{Distributional Cost-Effectiveness Analysis Based on Decision-Analytic Models}

DCEA is an intuitively appealing extension of conventional cost-effectiveness analysis (CEA) to quantify health equity impacts $[13,14]$. With a DCEA, the impact of the new intervention and standard of care on different social subgroups within the target patient population are estimated, which can 
be defined according to individual and non-individual factors such as race/ethnicity, socioeconomic status, geography, or a combination of factors. The distributions of health outcomes are compared in terms of total health (similar to a conventional CEA) as well as health inequality, taking into consideration the health opportunity costs. When the impacts of the new intervention on total health and health inequality are opposed, an equity trade-off analysis can help decide whether the new intervention is preferred over standard of care [14].

In practice, DCEA, much like CEA and other decisionanalytic approaches, relies on mathematical modelling that integrates different sources of evidence to estimate expected outcomes and opportunity costs by social subgroup with and without the new intervention. Mathematical modelling is typically required because all the information needed for decision making, including comparisons with all treatment alternatives for all important outcomes and sufficiently long follow-up durations, is rarely, if ever, available in a single empirical study. Even if such a study were practical, waiting for its results before making a decision is almost never an a priori-preferred option. By contrast, decision analysis based on mathematical modelling is an accepted and principled framework to make informed choices under uncertainty, organize and examine the impact of different factors, facilitate communication to stakeholders, and structure stakeholders' deliberations.

Figure 1 is an aducanumab-informed depiction of the concept of DCEA, with health equity impact evaluated across social subgroups defined according to race/ethnicity. Estimates were obtained by using aducanumab-specific relative treatment effects and age- and race-specific mild cognitive impairment prevalence and background progression rates in an open-source health economic model for AD [1, 15-19]. Uncertainty was incorporated with probabilistic sensitivity analysis. The analyses were performed in R [20], and the code is available online. The distribution of remaining lifetime QALYs expected with aducanumab and standard of care for patients with $\mathrm{AD}$ with mild cognitive impairment is presented (white bars), along with the corresponding net health benefits (NHBs) obtained by subtracting health opportunity costs at $\$$ US 100,000 per QALY shared equally among the population subgroups (grey bars).

Comparing the NHB distributions of aducanumab with standard of care, we can infer that the overall health is expected to increase with aducanumab when cost-effectively priced at $\$$ US10,000 per year but may result in an increase in the inequality in health outcomes between population subgroups defined according to race and ethnicity. We use inequality metrics to quantify how dissimilar the NHB is across social subgroups. Two such metrics are the Kolm inequality index, which measures dissimilarity in outcomes on an absolute scale [21], and the Atkinson inequality index, which works on a relative scale [22]. Figure 1 shows the 'efficiency-equity plane' with the joint uncertainty distribution of the incremental NHB (efficiency) and the difference in the Kolm inequality index of the NHB distributions across subgroups, the light grey upper point cloud on the equity-efficiency plane. To decide whether aducanumab is a worthwhile new intervention according to this analysis, we need to trade-off the gains in total health for worsening health inequality. If we are not concerned about inequity in health outcomes across the population subgroups, aducanumab is the strategy of choice when priced at $\$ \mathrm{US} 10,000$ per year. However, when we do care about health equity, the benefit will swing towards standard of care for increasing levels of inequality aversion, as depicted with the plot where the equally distributed equivalent incremental QALYs (i.e. the Atkinson index of social welfare on the QALY scale) are presented as a function of the Atkinson inequality aversion parameter, representing the degree of social preferences for reducing health inequities $[13,14]$. However, at the original intended aducanumab price of \$US56,000 per year, the health opportunity costs are greater than the benefits generated by aducanumab, and population health is lost on top of the worse health inequality metrics. (See the dark grey lower point cloud on the equity-efficiency plane in Fig. 1.) This conclusion is qualitatively similar, even at the latest proposed price of aducanumab, which is roughly half.

The above is a first-order exploration to illustrate DCEA. A more complete health equity impact evaluation of aducanumab would consider additional social subgroups across which we want to quantify health equity impact (e.g., age, gender, socioeconomic status, geographic location, or a combination of these factors) [23], race-specific amyloid positron emission tomography test performance to identify eligible patients, and anticipated test and treatment access and uptake by social subgroup. The findings of a DCEA can be sensitive to the assumed distribution of the opportunity costs across the social groups of interest. The assumption of equally shared opportunity costs in this example is convenient and arguably conservative but may not be realistic. However, determining appropriate distributions is a challenging and complex issue [24]. As such, a more comprehensive DCEA should assess the robustness of the health equity impact estimates with scenario analyses covering a range of values for the opportunity costs per QALY and their distribution over the social subgroups of interest, reflecting both public and private insurance programmes. Social distributions of disease prevalence and healthcare utilization can be a start to define how opportunity costs are allotted according to sex, race, and economic and insurance status, which can be further adjusted based on expert judgement [24]. 

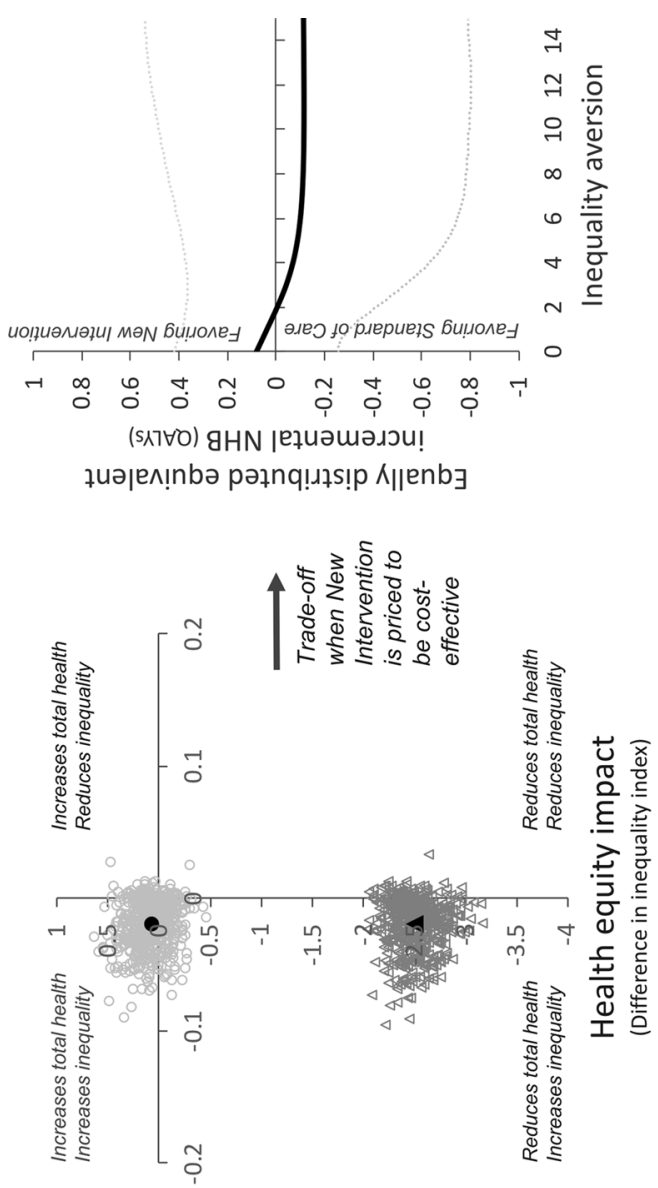

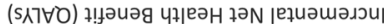

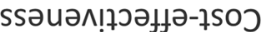

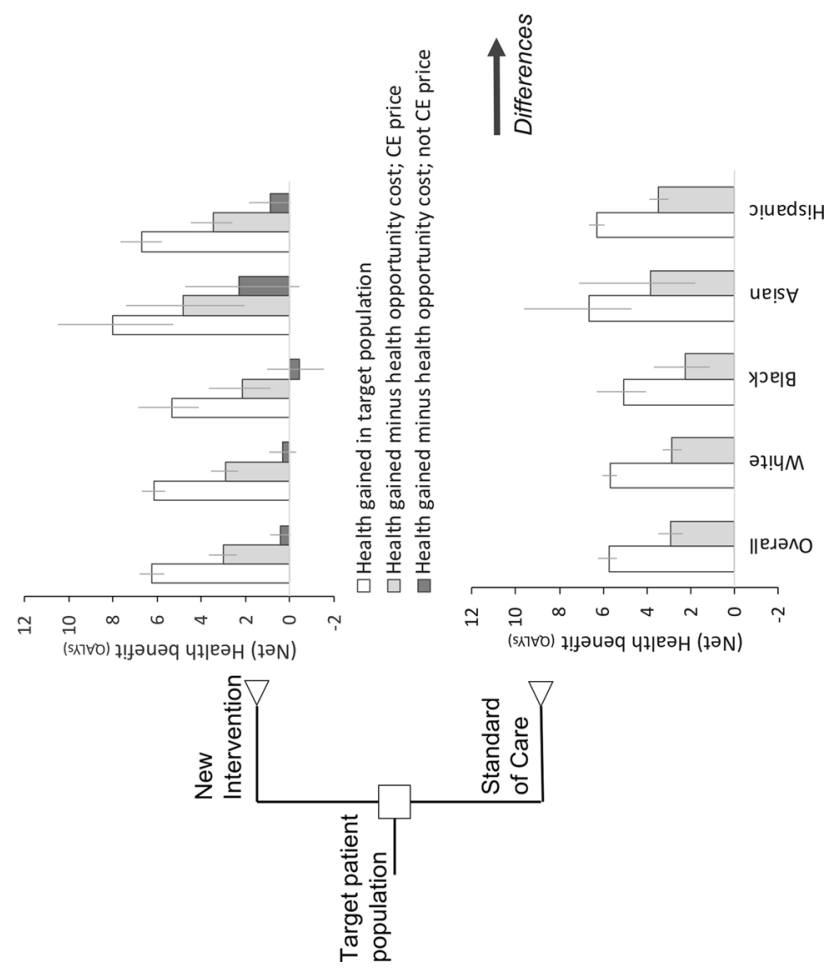

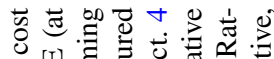

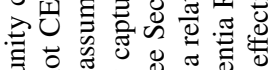

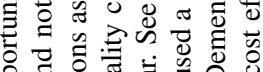
产完. Е $\widehat{0}$

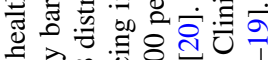

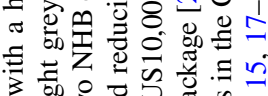

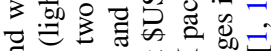
สี D.

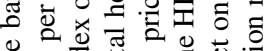
용 उद⿱

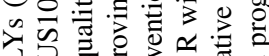

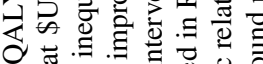

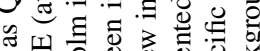

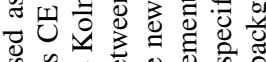

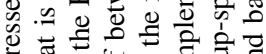

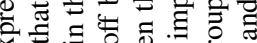

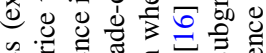

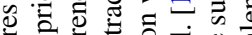

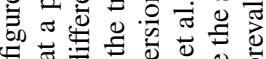

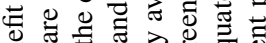

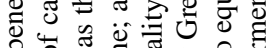

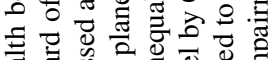
ब

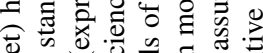

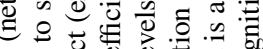

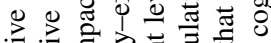

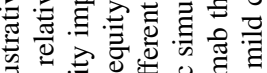

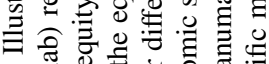

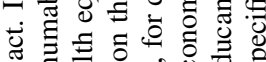

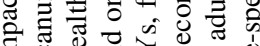

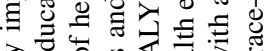

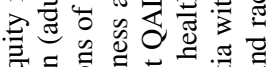
छี

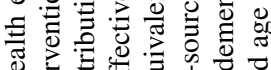

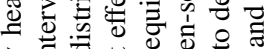

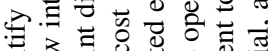

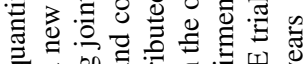

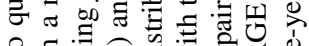
을 을 公 30

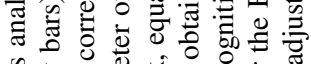

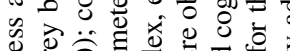

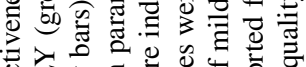

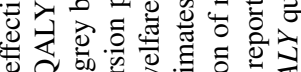

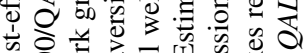

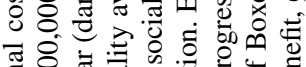

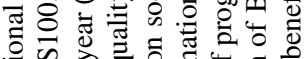

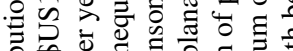

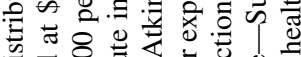
A뭉용

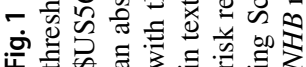




\section{A Key Evidence Gap for New Interventions}

The social subgroups of interest across which we quantify health equity impact (e.g., race/ethnicity, economic, geographic, or a combination) may constitute different distributions of age, sex, and race. Randomized controlled trials (RCTs) of new interventions are typically not designed to estimate treatment effects stratified by the patient characteristics of interest for a DCEA. The treatment effects of a new intervention for racial and ethnic minority groups are frequently uncertain because of limited clinical research participation. Only about $10 \%$ of the aducanumab trial participants were of Asian descent, and only six Black people were included in the high-dose arms [1]. Clearly, efforts to ensure that clinical trials of new interventions have a more representative and diverse study population are required and should address barriers at the system, individual, and interpersonal level [25]. However, such evidence gaps do not automatically render DCEA analyses moot, futile, or vacuous. Pursuing the analyses and propagating uncertainties throughout contributes to understanding how much we do not know and to more honest policy discussions. Employing a decision-modelling approach provides a powerful framework to evaluate, understand, and communicate the implications of the uncertainty in treatment effects for minority populations on health equity impact and estimates of value and for decision making. For example, we can calculate the probability that the new intervention will worsen health inequality and quantify the value of additional research to improve confidence in decision making with regards to health equity objectives.

\section{Some Comments on Corresponding Estimates from Evidence Sources to Model Parameters}

Many resources and tutorials for DCEA have been published in the last few years $[13,14,26]$. We add a few observations regarding the use of evidence to inform mathematicalmodel-based DCEAs of new interventions. If the estimates that are available to inform model parameters are obtained from population samples that differ from the target population of the DCEA in important ways, the estimated parameters' values will not transport (or transfer or generalize) to the DCEA context, and the results of the DCEA analysis will be 'externally biased' [27-29]. We expand on this challenge for different groups of parameters in a DCEA.

Most mathematical models estimate clinical and cost outcomes under a standard of care and then parameterize clinical and cost outcomes under alternative treatments using relative treatment effects. Ideally, parameters that pertain to the standard of care should be obtained from samples that resemble the target population in terms of the joint distribution of prognostic factors for outcomes, resource use, or costs, within each social subgroup and marginally over all social subgroups $[30,31]$. In practice, this implies that parameters for outcomes, resource use, and costs for the standard of care should be obtained specifically for the context of the DCEA from real-world data.

Parameter estimates for relative treatment effects of (the) new intervention(s) versus standard of care are typically obtained from RCTs. A DCEA would require relative treatment effects for each social subgroup; important differences in the distribution of effect modifiers between the RCT sample and the target population limit the generalizability of the estimates. Although there is no guarantee that the treatmenteffect modifiers will be the same variables as the prognostic factors for outcomes under the standard of care, empirically they are often fewer, or even a subset of the latter [32, 33]. This would imply that relative treatment-effect estimates for the new intervention need not be stratified to the same degree as the parameters for absolute outcomes with standard of care to be relevant for the social subgroups of interest.

Even when the important treatment-effect modifiers are known and measured, generalizing the RCT's treatmenteffect estimates to the DCEA's target population is challenging $[34,35]$. The most rigorous approaches require bespoke statistical analysis and access to individual patient data from the clinical trial and real-world data for the target population [35]. If such is not possible or practical, one is forced to use subgroup-specific treatment-effect estimates from the clinical trial and make assumptions to address their non-generalizability [29]. Because RCTs are typically not powered to estimate treatment effects in subgroups, social subgroupspecific treatment effects are typically imprecisely estimated. To increase the statistical precision of the typically imprecise estimates, one may need to assume exchangeable subgroup effects [36] and/or elicit pertinent information from experts and prior knowledge in the form of prior probability distributions that are included in the modelling analyses [29, 37, 38].

Finally, it is always a good idea to perform sensitivity analyses using alternative methods to estimate or predict relative treatment effects for the new intervention among minority populations when evidence is limited [27, 28, 31]. This reveals that the uncertainty in health equity impact estimates obtained with the model-based DCEA is larger than the propagated parameter uncertainty because it includes structural uncertainty as well. 


\section{Conclusion}

If we are serious about population-level decision making that not only is focused on improving total health but also aims to improve health equity, we should consider routinely assessing the health equity impact of new interventions and quantifying potential trade-offs. A practical approach is to augment the HTA of new interventions with DCEAbased health equity impact analyses [13, 14]. Gaps in the evidence base because of limited clinical research participation among racial and ethnic minority groups result in uncertainties about their treatment effects but do not preclude a DCEA. Understanding these uncertainties has implications for fair pricing and decision making and for future research. Specifically, for aducanumab in AD, a formal DCEA will quantify how its approval may impact on existing disparities in health outcomes given its efficacy, safety profile, costs, and data gaps and therefore provide us with a more complete picture of its value.

Acknowledgements Funding Drs Jansen and Phillips were supported by grants from the National Cancer Institute (NCI) of the NIH (R01 CA221870, R01 CA221870-S1) and from the National Human Genome Research Institute (R01HG011792); Dr Trikalinos was supported in part from an NCI grant (U01CA265750). The funders had no role in the preparation, review, or approval of the manuscript or the decision to submit the manuscript for publication.

Conflicts of Interest Dr Jansen is a part-time salaried employee of the Precision Medicine Group, where he provides methodological expertise for health economics and outcomes research studies unrelated to this paper. As part of his compensation, he received Precision Medicine Group stock options that have not been exercisable at the time of writing. Dr Trikalinos has no conflicts of interest related to the current manuscript. Dr Trikalinos is methodological consultant to Latham and Watkins and Pacira Pharmaceuticals on topics unrelated to this paper. Dr. Phillips receives consulting income from Illumina, Inc. and honoraria from participation on evidence review panels for the Institute for Clinical and Economic Review, outside of the submitted work.

Ethics approval Not applicable.

Availability of data and material The $\mathrm{R}$ code of the example analyses is available in a GitHub repository: https://github.com/jeroenpjansen/ Basic_DCEA.

Consent to participate Not applicable.

Consent for publication Not applicable.

Code availability The $\mathrm{R}$ code of the example analyses is available in a GitHub repository: https://github.com/jeroenpjansen/Basic_DCEA.

Author contributions All authors contributed to the drafting of the manuscript. JPJ performed the example analyses.

Open Access This article is licensed under a Creative Commons Attribution-NonCommercial 4.0 International License, which permits any non-commercial use, sharing, adaptation, distribution and reproduction in any medium or format, as long as you give appropriate credit to the original author(s) and the source, provide a link to the Creative Commons licence, and indicate if changes were made. The images or other third party material in this article are included in the article's Creative Commons licence, unless indicated otherwise in a credit line to the material. If material is not included in the article's Creative Commons licence and your intended use is not permitted by statutory regulation or exceeds the permitted use, you will need to obtain permission directly from the copyright holder. To view a copy of this licence, visit http://creativecommons.org/licenses/by-nc/4.0/.

\section{References}

1. Food and Drug Administration. Combined FDA and Applicant PCNS Drugs Advisory Committee Briefing Document. 2020.

2. Braveman P. Health disparities and health equity: concepts and measurement. Annu Rev Public Health. 2006;21(27):167-94.

3. James SA. Epidemiologic research on health disparities: some thoughts on history and current developments. Epidemiol Rev. 2009;31:1-6.

4. Penman-Aguilar A, Talih M, Huang D, Moonesinghe R, Bouye K, Beckles G. Measurement of health disparities, health inequities, and social determinants of health to support the advancement of health equity. Journal of public health management and practice: JPHMP. 2016;22(Suppl 1):S33.

5. Tan TQ, Kullar R, Swartz TH, Mathew TA, Piggott DA, Berthaud V. Location matters: geographic disparities and impact of coronavirus disease 2019. J Infect Dis. 2020;222(12):1951-4.

6. Tai DBG, Shah A, Doubeni CA, Sia IG, Wieland ML. The disproportionate impact of COVID-19 on racial and ethnic minorities in the United States. Clin Infect Dis. 2021;72(4):703-6.

7. Matthews KA, Xu W, Gaglioti AH, Holt JB, Croft JB, Mack D, McGuire LC. Racial and ethnic estimates of Alzheimer's disease and related dementias in the United States (2015-2060) in adults aged $\geq 65$ years. Alzheimers Dement. 2019;15(1):17-24.

8. Kawas CH, Corrada MM, Whitmer RA. Diversity and disparities in dementia diagnosis and care: a challenge for all of us. JAMA Neurol. 2021;2:2. https://doi.org/10.1001/jamaneurol.2021.0285.

9. World Health Organization. Health Systems. Equity https://www. who.int/health-topics/health-equity. Accessed December 16, 2021

10. Braveman P. A new definition of health equity to guide future efforts and measure progress. Health Aff. 2017;22:10.

11. Alvidrez J, Castille D, Laude-Sharp M, Rosario A, Tabor D. The national institute on minority health and health disparities research framework. Am J Public Health. 2019;109(S1):S16-20.

12. Vanness DJ, Lomas J, Ahn H. A health opportunity cost threshold for cost-effectiveness analysis in the United States. Ann Intern Med. 2021;174(1):25-32.

13. Asaria M, Griffin S, Cookson R. Distributional cost-effectiveness analysis: a tutorial. Med Decis Making. 2016;36(1):8-19.

14. Cookson R, Mirelman AJ, Griffin S, Asaria M, Dawkins B, Norheim OF, Verguet S, Culyer AJ. Using cost-effectiveness analysis to address health equity concerns. Value in Health. 2017;20(2):206-12.

15. Lin GA, Whittington MD, Synnott PG, McKenna A, Campbell J, Pearson SD, Rind DM. Aducanumab for Alzheimer's Disease: Effectiveness and Value; Evidence Report. Institute for Clinical and Economic Review, June 30, 2021. https://icer.org/assessment/ alzheimers-disease-2021/

16. Green C, Handels R, Gustavsson A, Wimo A, Winblad B, Sköldunger A, Jönsson L. Assessing cost-effectiveness of early intervention in Alzheimer's disease: An open-source modeling framework. Alzheimer's \& Dementia. 2019;15(10):1309-21. 
17. Michaud TL, Su D, Siahpush M, Murman DL. The risk of incident mild cognitive impairment and progression to dementia considering mild cognitive impairment subtypes. Dement Geriatr Cogn Disord Extra. 2017;7(1):15-29.

18. Hernandez Saucedo H, Whitmer RA, Glymour M, DeCarli C, Mayeda ER, Gilsanz P, Miles SQ, Bhulani N, Farias ST, Olichney $\mathrm{J}$, Mungas D. Measuring cognitive health in ethnically diverse older adults. J Gerontol B Psychol Sci Soc Sci. 2021;2:62.

19. U.S. Census Bureau. Table NC-EST2019-ASR5H Annual Estimates of the Resident Population by Sex, Age, Race Alone or in Combination, 1 and Hispanic Origin for the United States: April 1, 2010 to July 1, 2019. Retrieved from https://www.census.gov/ newsroom/press-kits/2020/population-estimates-detailed.html

20. Incerti D, Jansen JP. hesim: Health Economic Simulation Modeling and Decision Analysis. arXiv: 2102.09437 [stat.AP].

21. Kolm SC. Unequal inequalities. I. J Econ Theory. 1976;12(3):416-42.

22. Atkinson AB. On the measurement of inequality. J Econ Theory. 1970;2(3):244-63.

23. McRae J, Onukwugha E. Why the gap in evaluating the social constructs and the value of medicines? Pharmacoeconomics. 2021;30:1-8.

24. Love-Koh J. Health opportunity costs. In: Cookson R, Griffin S, Norheim OF, Culyer AJ, editors. Distributional cost-effectiveness analysis: Quantifying health equity impacts and trade-offs. Oxford University Press; 2020 Sep 30. p 174-194.

25. Hamel LM, Penner LA, Albrecht TL, Heath E, Gwede CK, Eggly S. Barriers to clinical trial enrollment in racial and ethnic minority patients with cancer. Cancer Control. 2016;23(4):327-37.

26. Cookson R, Griffin S, Norheim OF, Culyer AJ, editors. Distributional cost-effectiveness analysis: Quantifying health equity impacts and trade-offs. Oxford University Press; 2020.

27. Trikalinos TA, Russell LB, Sanders DG. Evidence synthesis for informing cost-effectiveness analysis. In: Cost-effectiveness in health and medicine. New York: Oxford University Press; 2017. p. 237-76.

28. Sanders GD, Neumann PJ, Basu A, Brock DW, Feeny D, Krahn M, Kuntz KM, Meltzer DO, Owens DK, Prosser LA,
Salomon JA. Recommendations for conduct, methodological practices, and reporting of cost-effectiveness analyses: second panel on cost-effectiveness in health and medicine. JAMA. 2016;316(10):1093-103.

29. Turner RM, Spiegelhalter DJ, Smith GC, Thompson SG. Bias modelling in evidence synthesis. J R Stat Soc Ser A (Stat Soc). 2009;172(1):21-47.

30. Neumann PJ, Kim DD, Trikalinos TA, Sculpher MJ, Salomon JA, Prosser LA, Owens DK, Meltzer DO, Kuntz KM, Krahn M, Feeny D. Future directions for cost-effectiveness analyses in health and medicine. Med Decis Making. 2018;38(7):767-77.

31. Dahabreh IJ, Trikalinos TA, Balk EM, Wong JB. Recommendations for the conduct and reporting of modeling and simulation studies in health technology assessment. Ann Intern Med. 2016;165(8):575-81.

32. Kent DM, Steyerberg E, van Klaveren D. Personalized evidence based medicine: predictive approaches to heterogeneous treatment effects. BMJ. 2018;10:363.

33. Kent DM, Nelson J, Dahabreh IJ, Rothwell PM, Altman DG, Hayward RA. Risk and treatment effect heterogeneity: re-analysis of individual participant data from 32 large clinical trials. Int J Epidemiol. 2016;45(6):2075-88.

34. Dahabreh IJ, Hernán MA. Extending inferences from a randomized trial to a target population. Eur J Epidemiol. 2019;34(8):719-22.

35. Robertson SE, Steingrimsson JA, Joyce NR, Stuart EA, Dahabreh IJ. Estimating subgroup effects in generalizability and transportability analyses. arXiv preprint arXiv: 2109.14075. 2021 Sep 28.

36. Henderson NC, Louis TA, Wang C, Varadhan R. Bayesian analysis of heterogeneous treatment effects for patient-centered outcomes research. Health Serv Outcomes Res Method. 2016;16(4):213-33.

37. Gosling JP. SHELF: the Sheffield elicitation framework. In Elicitation 2018 (pp. 61-93). Springer, Cham.

38. O'Hagan A. Expert knowledge elicitation: subjective but scientific. Am Stat. 2019;73(sup1):69-81. 